\title{
Borel Summability for a Nonpolynomial Potential
}

\author{
G. Auberson \\ Département de Physique Mathématique`, U.S.T.L., F-34060 Montpellier Cedex, France
}

\begin{abstract}
We consider the energy levels of a one-dimensional quantum system in the rational potential $\frac{1}{2} x^{2} \pm g x^{4} /\left(1+\alpha g x^{2}\right)$. Their perturbation expansions in $g$ are shown to be Borel summable. The proof is flexible enough to allow simple extensions to other nonpolynomial interactions.
\end{abstract}

\section{Introduction}

In recent years much attention has been devoted to the question of the Borel summability of perturbation expansions for quantities of interest in various quantum mechanical systems and quantum field models. Proofs of such a summability property have been given, e.g. for the energy levels of anharmonic oscillators [1] and for field-theoretic analogues, namely the Schwinger functions of super-renormalizable models $\left(\varphi_{2}^{4}\right.$ and $\varphi_{3}^{4}$ theories) [2]. The purpose of this paper is to investigate the Borel summability of the perturbation expansion of the energy levels in a one-dimensional model with a "singular" (nonpolynomial) potential. Specifically, we shall consider the Hamiltonian

$$
H^{ \pm}(g)=\frac{1}{2} p^{2}+V^{ \pm}(x ; g) \equiv \frac{1}{2} p^{2}+\frac{1}{2} x^{2} \pm \frac{g x^{4}}{1+\alpha g x^{2}}
$$

on the Hilbert space $L^{2}(-\infty, \infty)$, where the "physical" range of the parameters $g$ and $\alpha$ is

$$
\left.\begin{array}{l}
g \geq 0, \\
\alpha>0 \text { in the "+" case, } \\
\alpha>2 \text { in the " - " case }\left[\text { in order that } V^{-}(x ; g) \rightarrow+\infty \text { for } x^{2} \rightarrow \infty\right] .
\end{array}\right\}
$$

* Physique Mathématique et Théorique, Equipe de Recherche associée au CNRS 
It will be shown that the (asymptotic) perturbation expansion of the eigenvalues $E_{j}^{ \pm}(g)$ in $g$ at fixed $\alpha$

$$
E_{j}^{ \pm}(g) \sim \sum_{n=0}^{\infty} E_{j, n}^{ \pm} g^{n}
$$

is Borel summable to the true value $E_{j}^{ \pm}(g)$. Furthermore, it will clearly appear in the course of the proof that our method can be applied to other rational (or simply analytic) interactions.

As for the physical relevance of our choice of the specific interaction in Eq. (I.1), a few words are in order. The potential $V^{-}(x ; g)$ turns out to arise in a certain laser model after reduction of the Fokker-Planck equation to a Schrödinger equation [3]. Moreover, both perturbative [4] and nonperturbative [5] numerical calculations of the first energy levels have already been performed. Let us mention that each coefficient $E_{j, n}^{ \pm}$is a polynomial of degree $(n-1)$ in $\alpha$ with rational coefficients, as is most easily seen by using a recursive method of computation à la Bender and Wu [6] [see also Eqs. (III.9) and (III.10)]. A remark is appropriate at this point: in order to make practical perturbative calculations, the expansion in $g$ is not necessarily the best thing to use. For instance, in the large $\alpha$ regime, a calculation relying on an expansion in $g$ at fixed $\alpha g$ (which resorts to regular perturbation theory and is easily shown to have a nonzero convergence radius) would certainly be more efficient.

Our viewpoint is different: we are primarily interested in the perturbation with respect to the coupling constant $g$, because the interaction in Eq. (I.1) has the usual "field theoretic" form $V^{ \pm}(x ; g)=\mathscr{V}^{ \pm}\left(g x^{2}\right) / g$, which makes the series in powers of $g$ an expansion in terms of Feynman graphs collected according to their number of loops. In fact, our initial motivation for studying the Borel summability of the expansion (I.3) was in trying to understand some unexpected feature which arises when one looks for the asymptotics of the coefficients $E_{0, n}^{-}$of the fundamental level through the Lipatov (or instanton) approach [7]. We shall return to this point later on.

As far as singular perturbation theory is concerned, we want to emphasize the distinctive feature of the potential $V^{ \pm}(x ; g)$, when compared to the polynomial (e.g. anharmonic) or entire ones. What makes the expansion (I.3) a divergent series for all nonvanishing $g$ in the latter cases is the bad behaviour of the perturbation relative to the harmonic term when $x^{2} \rightarrow \infty$. In our case, the perturbation $g x^{4} /\left(1+\alpha g x^{2}\right)$ is not worse than $x^{2}$ at infinity. Rather, the singularity of $V^{ \pm}(x ; g)$ at $x^{2}=-1 / \alpha g$ is responsible for a singularity of $E_{j}^{ \pm}(g)$ at $g=0$ and for the divergence of the expansion (I.3) ${ }^{1}$.

We shall prove that $E_{j}^{ \pm}(g)$ is an analytic function of $g$ at least in the small sector $0<|g|<R_{j},|\arg g| \leqq \theta(\theta$ can be arbitrarily close to $\pi)$, and that the perturbation expansion (I.3) is Borel summable to that funtion there, in a sense which is made precise in Eqs. (III.31) and (III.33). Our proof relies on the singular perturbation theory as developed mainly by Kato [8] and Simon [9-11]. There are two

1 That this expansion for the ground state energy $E_{0}^{+}(g)$ actually diverges for all $g \neq 0, \alpha>0$ follows from the fact that the coefficients $E_{0, n}^{+}$are shown [7] to be larger in absolute value than the coefficients $\left.E_{0, n}^{+}\right|_{\alpha=0}$ corresponding to the well-known [6] pure anharmonic case. In the "- " case, this is no longer true, but there is still strong numerical evidence [7] for the divergence of the perturbation expansion 
additional technical complications, specific to a perturbation which is not a polynomial in the coupling constant: i) the "remainders" of the perturbation series to be kept under control involve a larger number of terms, ii) the so-called "quadratic estimates", which are of common use in the polynomial case [9-11], no longer apply. The first problem is easily dealt with, whereas the second one requires an "ad hoc" argument.

After some preparatory steps in Sect. II, all the relevant details of the proof are given in Sect. III. Our results and a number of problems to be solved are discussed in Sect. IV.

\section{Preliminaries}

We first notice that for all values of the parameters $g$ and $\alpha$ satisfying Eq. (I.2), the Hamiltonian $H^{ \pm}(g)$ is a self-adjoint operator on the domain $D\left(p^{2}\right) \cap D\left(x^{2}\right)$ with compact resolvent. It has a purely discrete spectrum $\left\{E_{j}^{ \pm}(g)\right\}$ with a nondegenerate, strictly positive ground state energy $E_{0}^{ \pm}(g)$. All this follows directly from standard theorems $[8,11 \mathrm{~b}]^{2}$.

For complex values of $g$, our strategy will be the following.

i) We first show that the $H^{ \pm}(g)$ are well-defined operators as long as $g$ keeps away from the negative real axis, and form in fact an analytic family in the technical sense of Kato $[8,11 \mathrm{~b}]$ (Lemma 1 below).

ii) In order to infer from that the announced analytic structure of $E_{j}^{ \pm}(g)$ in the neighborhood of $g=0$, we need a convergence property $H^{ \pm}(g) \rightarrow H(0)$ as $g \rightarrow 0$ $(\arg g \neq \pi)$ in the norm resolvent sense, which insures the stability of the eigenvalues of $H(0)$ (Lemmas 2 and 3).

iii) Then a basic estimate (Lemma 4) allows us to deduce that the perturbation expansion (I.3) is "strongly asymptotic" to $E_{j}^{ \pm}(g)$ (Theorem 1), and to conclude, via a Watson-like theorem, that this expansion is Borel summable to $E_{j}^{ \pm}(g)$ (Theorem 2).

Notice that, as a consequence of i), thanks to the Kato-Rellich theorem $[8,11 \mathrm{~b}], E_{j}^{ \pm}(g)$ is analytic also in some neighborhood of the positive real axis (origin excluded), although the perturbation expansion does not need to be Borel summable in this whole region.

We now proceed to establish the

Lemma 1. The operators $H^{ \pm}(g)$ defined in Eq.(I.1) with common domain $D \equiv D\left(p^{2}\right) \cap D\left(x^{2}\right)$ form an analytic family in the cut plane $\mathscr{C} \equiv \mathbb{C} \backslash(-\infty, 0]$. That is to say:

a) $H^{ \pm}(g)$ is closed $\forall g \in \mathscr{C}$,

B) $H^{ \pm}(g)$ has a nonempty resolvent set $\forall g \in \mathscr{C}$,

$\gamma$ ) for each $\varphi \in D, H^{ \pm}(g) \varphi$ is a vector-valued analytic function on $\mathscr{C}$.

Proof. Let us write $H^{ \pm}(g)$ in the form

$$
H^{ \pm}(g)=\frac{1}{2} p^{2}+\left(\frac{1}{2} \pm \frac{1}{\alpha}\right) x^{2} \mp \frac{x^{2}}{\alpha\left(1+\alpha g x^{2}\right)} .
$$

2 Of course, it is essential that the potential $V^{ \pm}(x ; g)$ be positive for all physical values (I.2) of the parameters 
Then the property $\alpha$ ) is obvious since $H^{ \pm}(g)$ appears as the sum of a closed operator and a bounded one, viz. the self-adjoint operator $h_{0}^{ \pm} \equiv \frac{1}{2} p^{2}+\left(\frac{1}{2} \pm \frac{1}{\alpha}\right) x^{2}$ with domain $D$ and the multiplicative operator $v^{ \pm}(g) \equiv \mp x^{2} / \alpha\left(1+\alpha g x^{2}\right)$ which is bounded (both point-wise and in norm) by $1 / \alpha^{2}|g|$ if $\operatorname{Re} g \geqq 0$ and by $1 / \alpha^{2}|\operatorname{Im} g|$ if $\operatorname{Re} g<0$.

Notice also that the adjoint of $H^{ \pm}(g)$ is $H^{ \pm}\left(g^{*}\right)$ with the same domain $D$.

Consider now

$$
R_{g}^{ \pm}(\lambda) \equiv\left(h_{0}^{ \pm}-\lambda\right)^{-1}\left[\mathbb{1}+v^{ \pm}(g)\left(h_{0}^{ \pm}-\lambda\right)^{-1}\right]^{-1} .
$$

For each $g \in \mathscr{C}$, this expression is clearly well-defined as a bounded operator if the ( $g$ dependent) constant $\lambda$ is suitably chosen. Indeed, the resolvent $\left(h_{0}^{ \pm}-\lambda\right)^{-1}$ of the harmonic oscillator Hamiltonian converges to zero in norm when $\lambda \rightarrow-\infty$, so that $\left\|v^{ \pm}(g)\left(h_{0}^{ \pm}-\lambda\right)^{-1}\right\|<1$ for $-\lambda$ large enough, and $\left[\mathbb{1}+v^{ \pm}(g)\left(h_{0}^{ \pm}-\lambda\right)^{-1}\right]^{-1}$ can be defined as a bounded operator by means of a Neumann series. Then $\left[H^{ \pm}(g)-\lambda\right] R_{g}^{ \pm}(\lambda)=R_{g}^{ \pm}(\lambda)\left[H^{ \pm}(g)-\lambda\right]=\mathbb{1}$ by direct computation, which means that $R_{g}^{ \pm}(\lambda)$ is nothing but the resolvent $\left[H^{ \pm}(g)-\lambda\right]^{-1}$ and entails property $\beta$ ).

Finally, property $\gamma$ ) is equivalent to the statement that

$$
\left(\psi, H^{ \pm}(g) \varphi\right)=\left(\psi, h_{0}^{ \pm} \varphi\right) \mp \frac{1}{\alpha} \int_{-\infty}^{\infty} d x \psi^{*}(x) \varphi(x) \frac{x^{2}}{1+\alpha g x^{2}}
$$

is a complex-valued analytic function on $\mathscr{C}$ for each $\varphi \in D$ and each $\psi \in L^{2}(-\infty, \infty)$. But this is obvious on account of the absolute convergence of the integral. q.e.d.

The previous lemma tells us nothing about the location of the spectrum of $H^{ \pm}(g)$ for nonreal positive values of $g$. We already know that the resolvent set $\varrho\left(H^{ \pm}(g)\right)$ of $H^{ \pm}(g)$ is not empty, but the proof above only shows the existence of negative $\lambda \in \varrho\left(H^{ \pm}(g)\right)$ which actually go to $-\infty$ when $g \rightarrow 0$. In order to establish the stability property which we alluded to before, we have to make sure that $\varrho\left(H^{ \pm}(g)\right)$ is not repelled to infinity as $g \rightarrow 0$ and enjoys itself some kind of stability. Such a guarantee is provided by the

Lemma 2. For any $g$ in the cut plane $\mathscr{C}$, the resolvent set $\varrho\left(H^{ \pm}(g)\right)$ contains the negative real axis $(-\infty, 0)$.

Proof. First, let us state an important property of the potential $V^{ \pm}(x ; g)$ which will play a crucial role also in the following. Consider the function

$$
W^{ \pm}\left(g x^{2}\right) \equiv \frac{1}{x^{2}} V^{ \pm}(x ; g) .
$$

Then, for any $\theta, 0 \leqq \theta<\pi$, the image $I^{ \pm}$of the set $\{-\infty<x<\infty, 0 \leqq \arg g \leqq \theta\}$ by this function is contained in a half-plane of the form $\operatorname{Re}\left(e^{i \omega} W^{ \pm}\right) \geqq \delta>0$ with $|\omega|<\frac{\pi}{2}$. In our case, we have also $\pm \operatorname{Im} W^{ \pm} \geqq 0$. In fact, a simple inspection of the function $W^{ \pm}(z)=\frac{1}{2} \pm z /(1+\alpha z)$ shows that $I^{ \pm}$consists of that inner part of the circle intersecting the real axis at the points $z=\frac{1}{2}$ and $z=\frac{1}{2} \pm \frac{1}{\alpha}$ through respective 
angles $\theta$ and $\pi-\theta$, which lies in the $\left\{\begin{array}{l}\text { upper } \\ \text { lower }\end{array}\right\}$ complex plane. Hence

$$
\left.\begin{array}{l} 
\pm \operatorname{Im} V^{ \pm}(x ; g) \geqq 0, \\
\operatorname{Re}\left[e^{i \omega^{ \pm}} V^{ \pm}(x ; g)\right] \geqq \delta^{ \pm} x^{2}, \quad \forall x, 0 \leqq \arg g \leqq \theta,
\end{array}\right\}
$$

with

$$
\begin{aligned}
& \omega^{ \pm}=0, \quad \delta^{ \pm}=\left(\frac{1}{2}-\frac{1}{2 \alpha}\right) \pm \frac{1}{2 \alpha} \quad \text { if } \quad 0 \leqq \theta \leqq \frac{\pi}{2}, \\
& \omega^{ \pm}= \pm\left(\frac{\pi}{2}-\theta\right), \quad \delta^{ \pm}=\left[\left(\frac{1}{2}-\frac{1}{2 \alpha}\right) \pm \frac{1}{2 \alpha}\right] \sin \theta \quad \text { if } \quad \frac{\pi}{2}<\theta<\pi .
\end{aligned}
$$

Now, let $g=|g| e^{i \theta}$ be given with $0 \leqq \theta<\pi$. We claim that when the vector $\varphi$ goes through $D$, the set of expectation values $\left(\varphi, H^{ \pm}(g) \varphi\right)$ is contained in a sector $S_{\theta}^{ \pm}$of the complex plane whose opening angle is less than $\pi$. Actually, Eqs. (II.5) imply, firstly:

$$
\pm \operatorname{Im}\left(\varphi, H^{ \pm}(g) \varphi\right)= \pm\left(\varphi, \operatorname{Im} V^{ \pm}(x ; g) \varphi\right) \geqq 0,
$$

and secondly, since $\cos \omega^{ \pm}>0$ :

$$
\begin{aligned}
\operatorname{Re}\left(\varphi, H^{ \pm}(g) \varphi\right) & \geqq\left(\varphi, \operatorname{Re} V^{ \pm}(x ; g) \varphi\right) \geqq \operatorname{tg} \omega^{ \pm}\left(\varphi, \operatorname{Im} V^{ \pm}(x ; g) \varphi\right) \\
& \geqq \operatorname{tg} \omega^{ \pm} \operatorname{Im}\left(\varphi, H^{ \pm}(g) \varphi\right),
\end{aligned}
$$

which define such a sector indeed [notice that, for the moment, we make no use of the fact that $\delta^{ \pm}$is strictly positive in Eq. (II.5)].

The same is true, of course, for $-\pi<\theta \leqq 0$ (with sign reversals). Then, due to the "sectorial" property we just derived for $H^{ \pm}(g)$, together with its closedness, it follows from a known theorem [12] that $\mathbb{C} \backslash S_{\theta}^{ \pm} \subset \varrho\left(H^{ \pm}(g)\right)$. Since, according to Eq. (II.8), $S_{\theta}^{ \pm}$does not intersect the negative real axis, we conclude that $(-\infty, 0) \subset \varrho\left(H^{ \pm}(g)\right)$. q.e.d.

Then comes the property of norm resolvent convergence.

Lemma 3. Let $\left\{E_{j}(0)\right\}$ be the spectrum $\left\{\frac{1}{2}, \frac{3}{2}, \frac{5}{2}, \ldots\right\}$ and $\varrho(H(0))$ the resolvent set $\mathbb{C} \backslash\left\{E_{j}(0)\right\}$ of $H(0)$. Then, for any $\theta, 0 \leqq \theta<\pi$

$$
\lim _{\substack{|g| \rightarrow 0 \\|\arg g| \leqq \theta}}\left\|\left[H^{ \pm}(g)-\lambda\right]^{-1}-[H(0)-\lambda]^{-1}\right\|=0 \quad \forall \lambda \in \varrho(H(0)),
$$

the convergence being uniform in $\lambda$ on compact subsets of $\varrho(H(0))$.

Proof. As a consequence of the resolvent formula $(H-\mu)^{-1}=(H-\lambda)^{-1}$ $\cdot\left[\mathbb{1}-(\mu-\lambda)(H-\lambda)^{-1}\right]^{-1}$ plus connectedness of $\varrho(H(0))$, the full conclusion of the lemma will follow if one can prove Eq. (II.9) for one $\lambda$ not in $\varrho(H(0))$ and not in $\varrho\left(H^{ \pm}(g)\right)$ as $g \rightarrow 0$. In view of Lemma 2 , it is therefore sufficient to establish Eq. (II.9) for some negative $\lambda$. Now, one has

$$
\begin{aligned}
{\left[H^{ \pm}(g)+\lambda\right]^{-1}-[H(0)+\lambda]^{-1} } & =\left[H^{ \pm}(g)+\lambda\right]^{-1}\left(\frac{\mp g x^{4}}{1+\alpha g x^{2}}\right)[H(0)+\lambda]^{-1} \\
& =\mp g\left\{\left[H^{ \pm}(g)+\lambda\right]^{-1} \frac{x^{2}}{1+\alpha g x^{2}}\right\}\left\{x^{2}[H(0)+\lambda]^{-1}\right\},
\end{aligned}
$$


where both factors in the last expression are bounded operators when $\lambda>0, g \in \mathscr{C}$ [this is obvious for the first one and readily seen for the second one, e.g. by using the eigenvectors of $H(0)$ as a basis for $\left.L^{2}(-\infty, \infty)\right]$. Hence, it is enough to show that

$$
\left\|\left[H^{ \pm}(g)+\lambda\right]^{-1}\left[x^{2} /\left(1+\alpha g x^{2}\right)\right]\right\|=\left\|\left[x^{2} /\left(1+\alpha g^{*} x^{2}\right)\right]\left[H^{ \pm}\left(g^{*}\right)+\lambda\right]^{-1}\right\|
$$

is uniformly bounded in the sector $|\arg g| \leqq \theta$ or, equivalently, that

$$
\left\|\frac{x^{2}}{1+\alpha g x^{2}}\left[H^{ \pm}(g)+\lambda\right]^{-1} \varphi\right\| \leqq C\|\varphi\| \quad \forall \varphi \in L^{2}(-\infty, \infty)
$$

for some $C$ independent of $g$. In fact, Eq. (II.11) needs to be proved only $\forall \varphi \in \mathscr{S}$, the Schwartz space of $C^{\infty}$ functions of fast decrease, since $\mathscr{S}$ is known to be dense in $L^{2}(-\infty, \infty)$. But this in turn is equivalent to

$$
\left\|\frac{x^{2}}{1+\alpha g x^{2}} \psi\right\| \leqq C\left\|\left[H^{ \pm}(g)+\lambda\right] \psi\right\| \quad \forall \psi \in \mathscr{S},
$$

provided that the image of $\mathscr{S}$ by $\left[H^{ \pm}(g)+\lambda\right]^{-1}$ is contained in $\mathscr{S}$. Such a property is true indeed, although we shall omit the proof here (it can be done, e.g. along the lines of [13]). Now, Eq. (II.12) means that

$$
\left(\psi,\left|\frac{x^{2}}{1+\alpha g x^{2}}\right|^{2} \psi\right) \leqq C^{2}\left(\psi,\left[H^{ \pm}(g)+\lambda\right]^{+}[H(g)+\lambda] \psi\right) \quad \forall \psi \in \mathscr{S},
$$

or, since $\left|1+\alpha g x^{2}\right|$ is bounded below uniformly in $x$ and $g$ :

$$
b x^{4} \leqq\left[\frac{1}{2} p^{2}+V^{ \pm *}(x ; g)+\lambda\right]\left[\frac{1}{2} p^{2}+V^{ \pm}(x ; g)+\lambda\right]
$$

for some positive $b$ independent of $g$, $|\arg g| \leqq \theta$. Here, the inequality has to be understood in the sense of positive operators from $\mathscr{S}$ to $\mathscr{S}$.

To prove Eq. (II.14), we start from the inequality

$$
\left[\frac{1}{2} p^{2}+V^{ \pm *}+\lambda-\eta\left(x^{2}+\lambda\right)\right]\left[\frac{1}{2} p^{2}+V^{ \pm}+\lambda-\eta^{*}\left(x^{2}+\lambda\right)\right] \geqq 0,
$$

which is obviously valid for all complex $\eta$ 's. By expanding, we find

$$
\begin{aligned}
\left(\frac{1}{2} p^{2}+V^{ \pm *}+\lambda\right)\left(\frac{1}{2} p^{2}+V^{ \pm}+\lambda\right) \geqq & -|\eta|^{2} x^{4}+2 \lambda\left(\operatorname{Re} \eta-|\eta|^{2}\right) x^{2}+\lambda^{2}\left(2 \operatorname{Re} \eta-|\eta|^{2}\right) \\
& +2\left(x^{2}+\lambda\right) \operatorname{Re}\left(\eta V^{ \pm}\right)+\lambda \operatorname{Re} \eta p^{2}+\frac{1}{2}\left(\eta^{*} p^{2} x^{2}+\eta x^{2} p^{2}\right) .
\end{aligned}
$$

Let us use the formal commutation relations [which we are allowed to do since everything in Eq. (II.16) operates on $\mathscr{S}]$ in order to recast the last term of the right hand side in a more convenient form:

$$
\begin{aligned}
\eta^{*} p^{2} x^{2}+\eta x^{2} p^{2} & =2 \operatorname{Re} \eta p x^{2} p+(p-2 \operatorname{Im} \eta x)^{2}-p^{2}-4(\operatorname{Im} \eta x)^{2}-2 \operatorname{Re} \eta \\
& \geqq p\left(2 \operatorname{Re} \eta x^{2}-1\right) p-4(\operatorname{Im} \eta x)^{2}-2 \operatorname{Re} \eta
\end{aligned}
$$

Thus

$$
\begin{aligned}
\left(\frac{1}{2} p^{2}+V^{ \pm}+\lambda\right)\left(\frac{1}{2} p^{2}+V^{ \pm}+\lambda\right) \geqq & -|\eta|^{2} x^{4}+2\left[\lambda\left(\operatorname{Re} \eta-|\eta|^{2}\right)-(\operatorname{Im} \eta)^{2}\right] x^{2} \\
& +\left[\lambda^{2}\left(2 \operatorname{Re} \eta-|\eta|^{2}\right)-\operatorname{Re} \eta\right]+2\left(\mathrm{x}^{2}+\lambda\right) \operatorname{Re}\left(\eta V^{ \pm}\right) \\
& +p\left[\operatorname{Re} \eta\left(x^{2}+\lambda\right)-\frac{1}{2}\right] p
\end{aligned}
$$


Now, let us choose $\eta=\varepsilon e^{i \omega^{ \pm}}$with $\varepsilon>0$ and $\omega^{ \pm}$given by Eqs. (II.6) (assuming $0 \leqq \arg g \leqq \theta$ ). Then $\operatorname{Re}\left(\eta V^{ \pm}\right) \geqq \varepsilon \delta^{ \pm} x^{2}$ according to Eqs. (II.5), so that

$$
\begin{aligned}
\left(\frac{1}{2} p^{2}+V^{ \pm *}+\lambda\right)\left(\frac{1}{2} p^{2}+V^{ \pm}+\lambda\right) \geqq & \varepsilon\left(2 \delta^{ \pm}-\varepsilon\right) x^{4} \\
& +2 \varepsilon\left[\lambda\left(\cos \omega^{ \pm}+\delta^{ \pm}-\varepsilon\right)-\varepsilon \sin ^{2} \omega^{ \pm}\right] x^{2} \\
& +\varepsilon\left[\lambda^{2}\left(2 \cos \omega^{ \pm}-\varepsilon\right)-\cos \omega^{ \pm}\right] \\
& +p\left[\varepsilon \cos \omega^{ \pm}\left(x^{2}+\lambda\right)-\frac{1}{2}\right] p .
\end{aligned}
$$

Since $\delta^{ \pm}>0, \cos \omega^{ \pm}>0$, it is now evident that for sufficiently small $\varepsilon$ and large $\lambda$ (both independent of $g$ !), all terms of the right hand side are positive and

$$
\left[\frac{1}{2} p^{2}+V^{ \pm *}(x ; g)+\lambda\right]\left[\frac{1}{2} p^{2}+V^{ \pm}(x ; g)+\lambda\right] \geqq \varepsilon\left[2 \delta^{ \pm}-\varepsilon\right] x^{4} \text {. q.e.d. }
$$

\section{Proof of the Borel Summability}

Equipped with Lemmas 1 and 3, we are entitled to apply standard asymptotic perturbation theory $[8,11 \mathrm{~b}]$, which tells us that the (nondegenerate!) eigenvalues $E_{j}(0)=j+\frac{1}{2}$ of $H(0) \quad(j=0,1,2, \ldots)$ are stable with respect to the perturbation $\pm g x^{4} /\left(1+\alpha g x^{2}\right)$. This means that i) for each $j$, there is a $R_{j}^{ \pm}$such that the spectrum of $H^{ \pm}(g)$ has exactly one point in the disk $\left|E-E_{j}(0)\right|<\frac{1}{2}$ when $|g|<R_{j}^{ \pm},|\arg g| \leqq \theta$ $(0 \leqq \theta<\pi)$, ii) this point $E_{j}^{ \pm}(g)$ is a nondegenerate eigenvalue of $H^{ \pm}(g)$. Moreover, the corresponding eigenvector $\Omega_{j}^{ \pm}(g)$ can be represented as

$$
\Omega_{j}^{ \pm}(g)=\frac{i}{2 \pi} \underset{\left|E-E_{j}(0)\right|=1 / 2}{\oint_{2}} d E\left[H^{ \pm}(g)-E\right]^{-1} \Omega_{j}(0)
$$

and converges to $\Omega_{j}(0)$ as $|g| \rightarrow 0,|\arg g| \leqq \theta$. As a by-product of ii) plus Lemma 1 , for any $\theta<\pi$, each $E_{j}^{ \pm}(g)$ is an analytic function in the sector $0<|g|<R_{j}^{ \pm},|\arg g| \leqq \theta$ (by the Kato-Rellich theorem $[8,11 \mathrm{~b}])^{3}$.

We can now write:

$$
E_{j}^{ \pm}(g)=\frac{\left(\Omega_{j}(0), H^{ \pm}(g) \Omega_{j}^{ \pm}(g)\right)}{\left(\Omega_{j}(0), \Omega_{j}^{ \pm}(g)\right)}=E_{j}(0) \pm g \frac{\left(\Omega_{j}(0), U(g) \Omega_{j}^{ \pm}(g)\right)}{\left(\Omega_{j}(0), \Omega_{j}^{ \pm}(g)\right)},
$$

where

$$
U(g)=\frac{x^{4}}{1+\alpha g x^{2}},
$$

and use formulas (III.1) and (III.2) as a starting point for the study of the perturbation expansion of $E_{j}^{ \pm}(g)$. In fact, it is sufficient to study separately the numerator and the denominator in Eq. (III.2): if the expansions of these two functions can be shown to be Borel summable, then according to general theorems [14], this property will be transmitted to $E_{j}^{ \pm}(g)$. Consider first the denominator :

$$
D(g)=(\Omega(0), \Omega(g))
$$

(we temporarily omit the superscripts \pm and the index $j$ ).

3 We stress that, because $R_{j}^{ \pm}$may depend on $\theta$, this does not imply that $E_{J}^{ \pm}(g)$ is analytic in a small cut disk $0<|g|<r_{j}^{ \pm}, \arg g \neq \pi$ 
Since $H(g)=H(0) \pm g U(g)$, in order to build up an expansion of $D(g)$ in powers of $g$, we have first to expand $\Omega(g)$ in powers of $U(g)$ by using the resolvent formula (II.10) :

$$
\begin{aligned}
\Omega(g)= & \sum_{n=0}^{N}(\mp g)^{n} \frac{i}{2 \pi} \oint_{|E-j-1 / 2|=1 / 2} d E R_{0}(E)\left[U(g) R_{0}(E)\right]^{n} \Omega(0) \\
& +(\mp g)^{N+1} \frac{i}{2 \pi} \oint_{|E-j-1 / 2|=1 / 2} d E R_{g}(E) \\
& \cdot\left[U(g) R_{0}(E)\right]^{N+1} \Omega(0), \quad N=0,1,2, \ldots
\end{aligned}
$$

(we have put $R_{g}(E) \equiv[H(g)-E]^{-1}$ ), and then to expand $U(g)$ itself in powers of $g$ :

$$
U(g)=\sum_{m=0}^{M}(-\alpha g)^{m} x^{4+2 m}+(-\alpha g)^{M+1} U_{M+1}(g), \quad M=0,1,2, \ldots,
$$

where

$$
U_{m}(g) \equiv \frac{x^{4+2 m}}{1+\alpha g x^{2}}
$$

By proceeding inductively on $n$, one easily deduces from Eq. (III.6) an expansion of $\left[U(g) R_{0}(E)\right]^{n}$ up to the order $g^{N-n}$, which is needed in Eq. (III.5):

$$
\begin{aligned}
& {\left[U(g) R_{0}(E)\right]^{n}=\sum_{r=0}^{N-n}(-\alpha g)^{r} \sum_{\substack{m_{\imath} \geqq 0 \\
m_{1}+\ldots+m_{n}=r}} x^{4+2 m_{1}} R_{0}(E) \ldots x^{4+2 m_{n}} R_{0}(E)} \\
& +(-\alpha g)^{N-n+1} \sum_{p=1}^{n}\left\{\sum_{\substack{m_{i} \geqq 0 \\
m_{1}+\ldots+m_{p}=N-n}} x^{4+2 m_{1}} R_{0}(E) \ldots\right. \\
& \left.\ldots x^{4+2 m_{p-1}} R_{0}(E) U_{m_{p}+1}(g) R_{0}(E)\left[U(g) R_{0}(E)\right]^{n-p}\right\} \text {. }
\end{aligned}
$$

Thus:

where the vectors

$$
\Omega(g)=\sum_{n=0}^{N} g^{n} \Omega_{n}+g^{N+1} R_{N+1}(g),
$$

$$
\begin{aligned}
\Omega_{n}= & (\mp 1)^{n} \sum_{r=0}^{n}( \pm \alpha)^{r} \sum_{\substack{m_{i} \geqq 0 \\
m_{1}+\ldots+m_{n-1}=r}} \frac{i}{2 \pi} \oint_{|E-j-1 / 2|=1 / 2} d E R_{0}(E) x^{4+2 m_{1}} R_{0}(E) \ldots \\
& \ldots x^{4+2 m_{n-r} R_{0}(E) \Omega(0)}
\end{aligned}
$$

are obviously well defined at each order $n$, and the remainder

$$
\begin{aligned}
R_{N+1}(g)= & (\mp 1)^{N+1}\left\{\sum_{n=0}^{N}( \pm \alpha)^{N+1-n} \sum_{p=1}^{n} \sum_{\substack{m_{1} \geqq 0 \\
m_{1}+\ldots+m_{p}=N-n}} \frac{i}{2 \pi} \oint_{|E-j-1 / 2|=1 / 2} d E\right. \\
& \cdot R_{0}(E) x^{4+2 m_{1}} R_{0}(E) \ldots \\
& \ldots x^{4+2 m_{p-1}} R_{0}(E) U_{m_{p}+1}(g) R_{0}(E)\left[U(g) R_{0}(E)\right]^{n-p} \\
& \left.+\frac{i}{2 \pi} \oint_{|E-j-1 / 2|=1 / 2} d E R_{g}(E)\left[U(g) R_{0}(E)\right]^{N+1}\right\} \Omega(0)
\end{aligned}
$$

has to be kept under control. 
Inserting Eq. (III.9) into Eq. (III.4), we find

$$
\left|D(g)-\sum_{n=0}^{N}\left(\Omega(0), \Omega_{n}\right) g^{n}\right| \leqq|g|^{N+1}\left\|R_{N+1}(g)\right\|
$$

[we choose the normalization $\|\Omega(0)\|=1$ ], so that our task amounts to bound $\left\|R_{N+1}(g)\right\|$ in the sector $|g|<R_{j}$, $|\arg g| \leqq \theta$. First of all, the resolvent $R_{g}(E)$ is uniformly bounded on the product of this sector with the circle $|E-j-1 / 2|=1 / 2$ as a consequence of Lemma 3. Thus, Eq. (III.1) implies

$$
\begin{aligned}
& \left\|R_{N+1}(g)\right\| \leqq C_{1} \sum_{n=0}^{N} \alpha^{N+1-n} \sum_{p=1}^{n} \sum_{\substack{m_{i} \geqq 0 \\
m_{1}+\ldots+m_{p}=N-n}} \sup _{|E-j-1 / 2|=1 / 2} \\
& \quad \cdot\left\|\left[x^{4+2 m_{1}} R_{0}(E)\right] \ldots\left[x^{4+2 m_{p-1}} R_{0}(E)\right] \times\left[U_{m_{p}+1}(g) R_{0}(E)\right]\left[U(g) R_{0}(E)\right]^{n-p} \Omega(0)\right\| \\
& \quad+C_{2} \sup _{|E-j-1 / 2|=1 / 2}\left\|\left[U(g) R_{0}(E)\right]^{N+1} \Omega(0)\right\| .
\end{aligned}
$$

In this expression, the factors between brackets are not bounded operators [although $\Omega(0)$ is of course in the domain of their products]. We would like however to "factorize the norm" in order to get a more useful estimate. This can be achieved by means of the following trick. We transform each bracket into a bounded operator by inserting suitable powers of $x^{2}$ both at the right and at the left, in such a way that these powers cancel out between two successive factors. Of course, in so doing an overall power of $x^{2}$ will be pushed in front of $\Omega(0)$, but this is harmless since $\Omega(0)$ (an exponentially decreasing Hermite function) is in the domain of any power of $x$. More precisely, we rewrite the last term of Eq. (III.13) in the form

$$
\begin{aligned}
& \left\|\left[U(g) R_{0}(E)\right]^{N+1} \Omega(0)\right\| \\
& \left.=\| \prod_{n=1}^{N+1}\left[U(g)\left((c(n-1))^{2(n-1)}+x^{4(n-1)}\right)^{1 / 2} R_{0}(E)((c n))^{2 n}+x^{4 n}\right)^{-1 / 2}\right] \\
& \quad\left\{(c(N+1))^{2(N+1)}+x^{4(N+1)}\right\}^{1 / 2} \Omega(0) \|
\end{aligned}
$$

where the $n$-dependence of the constant term $(c n)^{2 n}$ has been so chosen as to optimize the forthcoming bounds. We now anticipate the fact (to be proven below) that for suitable $c$, the new factors between brackets are bounded operators, and deduce

$$
\begin{aligned}
& \left\|\left[U(g) R_{0}(E)\right]^{N+1} \Omega(0)\right\| \\
& \quad \leqq \prod_{n=1}^{N+1}\left\|U(g)\left((c(n-1))^{2(n-1)}+x^{4(n-1)}\right)^{1 / 2} R_{0}(E)\left((c n)^{2 n}+x^{4 n}\right)^{-1 / 2}\right\| \omega_{N+1},
\end{aligned}
$$

where

$$
\left.\omega_{N} \equiv \|[c N)^{2 N}+x^{4 N}\right]^{1 / 2} \Omega(0) \|
$$


Since for all $x,|U(g)| \leqq d_{g} x^{4}$ with

we obtain

$$
d_{g} \equiv \begin{cases}1 & \text { if } \quad|\arg g| \leqq \frac{\pi}{2}, \\ 1 / \sin |\arg g| & \text { if } \quad \frac{\pi}{2}<|\arg g| \leqq \theta,\end{cases}
$$

$$
\left\|\left[U(g) R_{0}(E)\right]^{N+1} \Omega(0)\right\| \leqq\left(d_{g}\right)^{N+1} \omega_{N+1} \prod_{n=1}^{N+1}\left\|M_{0, n}(E)\right\|,
$$

where $M_{0, n}(E)$, and more generally $M_{m, n}(E)$, is defined by

$$
\begin{aligned}
M_{m, n}(E) \equiv & x^{4+2 m}\left[(c(n-1))^{2(n-1)}+x^{4(n-1)}\right]^{1 / 2} \\
& \cdot R_{0}(E)\left[(c(n+m))^{2(n+m)}+x^{4(n+m)}\right]^{-1 / 2}, \\
& m=0,1, \ldots ; \quad n=1,2, \ldots
\end{aligned}
$$

(with the convention $0^{0}=0$ ).

In a completely similar way, using $\left|U_{m}(g)\right| \leqq d_{g} x^{4+2 m}$, we can obtain for the other terms of Eq. (III.13) bounds involving only the $M_{m, n}(E)$ :

$$
\begin{aligned}
& \left\|\left[x^{4+2 m_{1}} R_{0}(E)\right] \ldots\left[x^{4+2 m_{p-1}} R_{0}(E)\right]\left[U_{m_{p}+1}(g) R_{0}(E)\right]\left[U(g) R_{0}(E)\right]^{n-p} \Omega(0)\right\| \\
& \leqq\left(d_{g}\right)^{n-p+1} \omega_{N+1} \prod_{i=1}^{p-1}\left\|M_{m_{\imath}, m_{1}+\ldots+m_{i-1}+i}(E)\right\| \\
& \cdot\left\|M_{m_{p}+1, m_{1}+\ldots+m_{p-1}+p}(E)\right\| \prod_{q=N-n+p+1}\left\|M_{0, q+1}(E)\right\| .
\end{aligned}
$$

It remains to majorize the $M_{m, n}(E)$ themselves. This will be done on the basis of the following estimate, which constitutes the crucial ingredient of our method.

Lemma 4. There are constants $a$ and $c$ depending only on $j$ (not on $m$ and $n$ ) such that for all $m=0,1, \ldots, n=1,2, \ldots$ and $E,|E-j-1 / 2|=1 / 2$ :

$$
\begin{aligned}
& {[H(0)-E]^{+}\left[(c(n+m))^{2(n+m)}+x^{4(n+m)}\right][H(0)-E]} \\
& \quad \geqq a^{2} x^{8+4 m}\left[\left(c(n-1)^{2(n-1)}+x^{4(n-1)}\right],\right.
\end{aligned}
$$

where the inequality has to be taken in the sense of positive operators on the Schwartz space $\mathscr{S}$.

The proof is postponed to the appendix.

Exactly as in the proof of Lemma $3([H(0)-E]$ is a bijection from $\mathscr{S}$ to $\mathscr{S})$, we immediately infer from Eq. (III.21) that $\|\varphi\| \geqq a\left\|M_{m, n}(E) \varphi\right\|$ for all $\varphi \in \mathscr{S}$, thus for all $\varphi \in L^{2}(-\infty, \infty)$ by continuity, and conclude that $M_{m, n}(E)$ is a bounded operator with $(m, n)$-independent norm:

$$
\left\|M_{m, n}(E)\right\| \leqq \frac{1}{a}, \quad m=0,1, \ldots ; \quad n=1,2, \ldots .
$$

Using this in Eqs. (III.18) and (III.20) and returning to Eq. (III.13), we get:

$$
\begin{aligned}
\left\|R_{N+1}(g)\right\| \leqq & C_{1} \sum_{n=0}^{N} \alpha^{N+1-n} \sum_{p=1}^{n} 2^{N-n+p}\left(d_{g}\right)^{n-p+1} \\
& \cdot \omega_{N+1}\left(\frac{1}{a}\right)^{n}+C_{2} \omega_{N+1}\left(\frac{d_{g}}{a}\right)^{N+1}
\end{aligned}
$$


because the sum over the $m_{i}$ 's contains $(N-n+p-1) ! /(N-n) !(p-1) !<2^{N-n+p}$ terms. Then, since $a<1, d_{g} \geqq 1$ :

$$
\begin{aligned}
\left\|R_{N+1}(g)\right\| & \leqq C_{1} \omega_{N+1}\left(\frac{2 d_{g}}{a}\right)^{N} \sum_{n=0}^{N} \alpha^{N+1-n} \frac{1}{2^{n}} \sum_{p=1}^{n} 2^{p}+C_{2} \omega_{N+1}\left(\frac{d_{g}}{a}\right)^{N+1} \\
& \leqq C_{3} \omega_{N+1}\left(\frac{2 d_{g}}{a}\right)^{N+1} \sum_{q=0}^{N+1} \alpha^{q} \\
& \leqq C_{4} \omega_{N+1} s^{N+1}
\end{aligned}
$$

where

$$
S= \begin{cases}\frac{2 d_{g}}{a}, & \alpha<1, \\ \frac{2 d_{g}}{a} \alpha, & \alpha>1 .\end{cases}
$$

Only $\omega_{N}$ defined in Eq. (III.16) remains to be estimated.

One has:

$$
\omega_{N}=\left[(c N)^{2 N}+\left\|x^{2 N} \Omega(0)\right\|^{2}\right]^{1 / 2},
$$

and a straightforward evaluation (e.g. using the creator-annihilator formalism) gives :

$$
\left\|x^{M} \Omega_{j}(0)\right\|^{2}<2^{M} \frac{(M+j) !}{j !} \leqq 2^{j}(4 M)^{M},
$$

so that (assuming $c \geqq 8$ ):

$$
\omega_{N} \leqq \operatorname{const}(c N)^{N} \leqq \operatorname{const} \Gamma(N+1 / 2)(e c)^{N} .
$$

Inserting Eqs. (III.24) and (III.28) into Eq. (III.12), we finally obtain:

$$
\begin{gathered}
\left|D(g)-\sum_{n=0}^{N}\left(\Omega(0), \Omega_{n}\right) g^{n}\right| \leqq A \Gamma\left(N+\frac{3}{2}\right)(e c s)^{N+1}|g|^{N+1} \\
N=0,1,2, \ldots
\end{gathered}
$$

In exactly the same way one can show that the numerator $N(g) \equiv(\Omega(0), U(g) \Omega(g))$ in Eq. (III.2) fulfills the conditions :

$$
\begin{gathered}
\left|N(g)-\sum_{n=0}^{N}\left(\Omega(0), \bar{\Omega}_{n}\right) g^{n}\right| \leqq \bar{A} \Gamma\left(N+\frac{3}{2}\right)(e c s)^{N+1}|g|^{N+1} \\
N=0,1,2, \ldots
\end{gathered}
$$

(with the same $c$ and $s$ ) for suitably defined vectors $\bar{\Omega}_{n}$. Now, according to general properties (Theorems 3 and 6 of [14]; see also [11]), a rational combination like (III.2) of two functions $N(g)$ and $D(g)$ obeying the "strong asymptotic" conditions (III.29) and (III.30) obeys itself these conditions, with the same parameter ecs. This leads us to our main result:

Theorem 1. Let $\theta$ be given, $0<\theta<\pi$. Then each energy level $E_{j}^{ \pm}(g)$ of the Hamiltonian (I.1) is analytic in a sector $0<|g|<R_{j}^{ \pm},|\arg g| \leqq \theta$, and its perturbation 
expansion is "strongly asymptotic" there:

where

$$
\begin{gathered}
\left|E_{j}^{ \pm}(g)-\sum_{n=0}^{N} E_{j, n}^{ \pm} g^{n}\right| \leqq A_{j}^{ \pm} \Gamma\left(N+\frac{3}{2}\right)\left(\sigma_{j} d_{g}\right)^{N+1}|g|^{N+1}, \\
N=0,1,2, \ldots,
\end{gathered}
$$

$$
d_{g}= \begin{cases}1, & |\arg g| \leqq \frac{\pi}{2}, \\ \frac{1}{\sin |\arg g|}, & \frac{\pi}{2}<|\arg g| \leqq \theta\end{cases}
$$
On account of Eq. (III.25), we have $\sigma_{j}=2 e \frac{c_{j}}{a_{j}} \operatorname{Max}(\alpha, 1)$ with the estimates (A.8)
and (A.15) for $a_{j}$ and $c_{j}$.

In particular, Eq. (III.31) immediately implies

$$
\left|E_{j, n}^{ \pm}\right| \leqq A_{j}^{ \pm} \Gamma\left(n+\frac{1}{2}\right)\left(\sigma_{j}\right)^{n}, \quad n=0,1,2, \ldots
$$

An investigation à la Lipatov (as well as a numerical one) [7] reveals that the actual coefficients $E_{0, n}^{ \pm}$exactly saturate the bound (III.32) in the limit $n \rightarrow \infty$, although the "true" constant $\sigma_{0}$ is (as expected) considerably less than an estimation based on Eqs. (A.8) and (A.15), which gives $\sigma_{0}=700 \operatorname{Max}(\alpha, 1)$.

As for the Borel summability of the perturbation expansion, the full content of Theorem 1 [not only Eq. (III.32)] is needed in order to establish that $\sum_{n} E_{j, n}^{ \pm} g^{n}$ is Borel summable to the true function $E_{j}^{ \pm}(g)$. Actually, such a property then follows from Watson's theorem [15]. More precisely, within the notation of [14], Eq. (III.31) just means that $E_{j}^{ \pm}(g)$ belongs to " $W$ " classes of Borel summable functions :

$$
E_{j}^{ \pm}(g) \in W-\left(\theta-\frac{\pi}{2}, R_{j}^{ \pm}, \sigma_{j}\right) \text { for all } \theta, \quad \frac{\pi}{2}<\theta<\pi .
$$

Hence, Theorem 2 of that reference directly applies and allows us to conclude:

Theorem 2. The Borel transform $\tilde{E}_{j}^{ \pm}(t)=\sum_{n=0}^{\infty} \frac{1}{n !} E_{j, n}^{ \pm} t^{n}$ (holomorphic in the disk $\left.|t|<1 / \sigma_{j}\right)$ has an analytic continuation to the half-plane $\operatorname{Re} t>0$, and is bounded there by:

$$
\left|\tilde{E}_{j}^{ \pm}(t)\right| \leqq B_{j}^{ \pm} e^{|t| / R_{j}^{ \pm}} \quad \forall t, \quad|\arg t| \leqq \theta
$$

for any $\theta<\frac{\pi}{2}\left(B_{j}^{ \pm}\right.$and $R_{j}^{ \pm}$may depend on $\theta$ ).

As is well known, this theorem in turn provides a justification for possible summation procedures of the perturbation series based on the inversion formula $E_{j}^{ \pm}(g)=\int_{0}^{\infty} d t e^{-t} \tilde{E}_{j}^{ \pm}(t g)$.

\section{Discussion}

We have shown that each eigenvalue $E_{j}^{ \pm}(g)$ of the nonpolynomial Hamiltonian (I.1) admits a perturbation expansion which is not only asymptotic, but even 
strongly asymptotic, and therefore Borel summable to $E_{j}^{ \pm}(g)$. In particular, we have not observed any difference at this level between the "+" case and the "- " case. It turns out that if one tries to improve the rigorous estimates (III.32) by looking for the asymptotics of the perturbation coefficients $E_{0, n}^{ \pm}$through the Lipatov method, such a similarity disappears [7]: in one case $(+)$ one finds a genuine instanton (and derives a reliable large order behaviour), in the other one $(-)$, there is no instanton at all (and a modified functional technique has to be devised in order to get the large $n$ behaviour). Such a phenomenon, which is likely to occur for many rational interactions, remains to be understood.

Considering now the analyticity domain of the Borel transform $\tilde{E}_{j}^{ \pm}(t)$ (Theorem 2), we have no indication that it can be significantly enlarged. Actually, the possibility of an extension to a sector of opening angle larger than $\pi$ [which would require the analytic continuation of $E_{j}^{ \pm}(g)$ to a "second sheet" of the complex $g$-plane in the vicinity of the origin] is not supported by numerical analysis [16]. Instead, the latter suggests rather a domain of the form $\operatorname{Re} t>-1 / \sigma_{j}$. As to whether the constant $R_{j}^{ \pm}$in Theorem 2 can be pushed to infinity (this in particular would allow the Borel summation to apply for all positive $g$ ), it amounts to whether $E_{j}^{ \pm}(g)$ is actually analytic in the whole cut plane. We have made no attempt in this work to investigate such global analytic properties of $E_{j}^{ \pm}(g)$. In this respect, let us notice that the method used by Loeffel and Martin [17] in the anharmonic case, which essentially consists in controlling the motion of the zeros of the wave function, cannot be applied in a straightforward manner to our case (the power of the Symanzik scaling argument is partly lost).

One easy result is worth mentioning however. We assert that the function $E_{0}^{-}(g)$ necessarily has complex singularities in the cut $g$-plane, at least for a certain range of values of the parameter $\alpha$. This can be seen through the following argument, which uses as an additional input the (readily found) expressions of the first three coefficients: $E_{0,1}^{-}=-3 / 4, \quad E_{0,2}^{-}=-21 / 8+(15 / 8) \alpha, \quad E_{0,3}^{-}=-333 / 16$ $+(45 / 2) \alpha-(105 / 16) \alpha^{2}$. Assume that $E_{0}^{-}(g)$ can be continued analytically to the whole cut plane. Then, the resolvent $R_{g}^{-}(\lambda)=\left[H^{-}(g)-\lambda\right]^{-1}$ being a compact operator [an easy consequence of Eq. (II.2)], this analytic continuation is still an eigenvalue of $H^{-}(g)$, according to a known theorem [18]. Hence, due to the positivity property $-\operatorname{Im} V^{-}(x ; g) / \operatorname{Im} g \geqq 0$ [Eq. (II.5)], it follows that $-E_{0}^{-}(g)$ is a Herglotz function. But $E_{0}^{-}(g)-E_{0}^{-}(\infty)$ is also a Stieltjes function, as a consequence of Theorem 1 and a simple perturbative argument in $1 / \mathrm{g}$. Therefore, the coefficients $(-1)^{n} E_{0, n}^{-} \quad(n=1,2, \ldots)$ are the moments of a positive measure $\left(-\frac{1}{\pi} \operatorname{Im} E_{0}^{-}(-|g|+i \varepsilon)\right)$ and must satisfy (amongst other conditions) the inequality $\left(E_{0,2}^{-}\right)^{2} \leqq E_{0,1}^{-} E_{0,3}^{-}$. Inserting the expressions given above, we find that this inequality is violated for $2.28 \leqq \alpha \leqq 2.74$, and conclude that our assumption cannot be correct in this range. ${ }^{4}$

Finally, a few words regarding the proof of Borel summability are in order. Our method relies on two main ingredients : Lemmas 2 and 3, the validity of which requires some geometrical properties of the range of values of the potential $V(x ; g)$,

4 Such a result implies that the direct use of Padé approximants in order to sum up the perturbation expansion of $E_{0}^{-}(g)$ cannot be put on firm grounds 
and the inequalities (III.21), which apply as soon as the potential, as well as its derivatives, enjoy some polynomial boundedness properties. Clearly enough, both ingredients are quite flexible in this form and, after suitable adjustments, they should permit the treatment of other rational (or even more general) potentials, including those involving more than one degree of freedom. In this way one can hope to apply our method to a large class of (properly restricted) interactions.

\section{Appendix}

Proof of Lemma 4. Given $H_{0} \equiv H(0)=\frac{1}{2} p^{2}+\frac{1}{2} x^{2}$ and $E=j+1 / 2+\frac{1}{2} e^{i \beta}$, let us first establish the two inequalities:

$$
\begin{aligned}
& \left(H_{0}-E^{*}\right)\left(H_{0}-E\right) \geqq \frac{1}{4}, \\
& \left(H_{0}-E^{*}\right)\left(H_{0}-E\right) \geqq \frac{a^{2}}{4} x^{4} \quad \text { for some }(j \text { dependent }) \quad a>0 .
\end{aligned}
$$

The first one is an immediate consequence of the spectral theorem:

$$
\left(H_{0}-E^{*}\right)\left(H_{0}-E\right)=\sum_{k=0}^{\infty}|k+1 / 2-E|^{2} P_{k} \geqq \frac{1}{4} \sum_{k=0}^{\infty} P_{k}=\frac{1}{4} \quad \forall j
$$

[where $P_{k}$ denotes the orthogonal projector onto $\Omega_{k}(0)$ ].

As for the second one, we have

$$
\begin{aligned}
\left(H_{0}-E^{*}\right)\left(H_{0}-E\right) & =H_{0}^{2}-2(j+1) H_{0}+j^{2}+1+(1-\cos \beta)\left(H_{0}-1 / 2\right)+j(1+\cos \beta) \\
& \geqq H_{0}^{2}-2(j+1) H_{0}+j^{2}+1,
\end{aligned}
$$

since $H_{0} \geqq \frac{1}{2}$. On the other hand

$$
H_{0}^{2}=\frac{1}{4} x^{4}+\frac{1}{4} p^{4}-\frac{1}{2}+\frac{1}{2} p x^{2} p \geqq \frac{1}{4} x^{4}+\frac{1}{4} p^{4}-\frac{1}{2},
$$

so that

$$
\begin{aligned}
\left(H_{0}-E^{*}\right)\left(H_{0}-E\right) & \geqq \frac{1}{4} x^{4}-(j+1) x^{2}-2 j-\frac{1}{2}+\left[\frac{1}{2} p^{2}-(j+1)\right]^{2} \\
& \geqq \frac{1}{4} x^{4}-(j+1) x^{2}-2 j-\frac{1}{2} .
\end{aligned}
$$

Thus, for any $u, 0<u<1$

$$
\begin{aligned}
\left(H_{0}-E^{*}\right)\left(H_{0}-E\right) & \geqq \frac{u}{4} x^{4}+\frac{1}{4}\left[\sqrt{1-u} x^{2}-2 \frac{j+1}{\sqrt{1-u}}\right]^{2}-\left[\frac{(j+1)^{2}}{1-u}+2 j+\frac{1}{2}\right] \\
& \geqq \frac{u}{4} x^{4}-\left[\frac{(j+1)^{2}}{1-u}+2 j+\frac{1}{2}\right] 4\left(H_{0}-E^{*}\right)\left(H_{0}-E\right),
\end{aligned}
$$

where we have used inequality (A.1) as a "feedback". Hence we obtain Eq. (A.2) with

$$
a^{2}=\sup _{0<u<1} \frac{u}{1+4\left[\frac{(j+1)^{2}}{1-u}+2 j+\frac{1}{2}\right]}
$$


Consider now the left hand side of Eq. (III.21). Because of Eq. (A.2) we can write

$$
\left(H_{0}-E^{*}\right)\left[(c q)^{2 q}+x^{4 q}\right]\left(H_{0}-E\right) \geqq \frac{a^{2}}{4}(c q)^{2 q} x^{4}+\left(H_{0}-E^{*}\right) x^{4 q}\left(H_{0}-E\right)
$$

Furthermore

$$
(q=n+m) \text {. }
$$

$$
\begin{aligned}
\left(H_{0}-E^{*}\right) x^{4 q}\left(H_{0}-E\right)= & \left(\frac{1}{2} p^{2}-E^{*}\right) x^{4 q}\left(\frac{1}{2} p^{2}-E\right)+\frac{1}{2} p x^{4 q+2} p \\
& +\frac{1}{4} x^{4 q+4}-(\operatorname{Re} E) x^{4 q+2}-\frac{1}{4}(4 q+1)(4 q+2) x^{4 q},(\text { A. } 10)
\end{aligned}
$$

and, using $\operatorname{Re} E \leqq j+1, q \geqq 1$ :

$$
\left(H_{0}-E^{*}\right) x^{4 q}\left(H_{0}-E\right) \geqq \frac{1}{4} x^{4 q+4}-(j+1) x^{4 q+2}-\frac{15}{2} q^{2} x^{4 q} .
$$

We then get rid of the middle term by inserting the inequality $x^{2} \leqq \frac{1}{2}\left(\varrho x^{4} / q^{2}+q^{2} / \varrho\right)$, valid for any $\varrho>0$ :

$$
\begin{aligned}
\left(H_{0}-E^{*}\right) x^{4 q}\left(H_{0}-E\right) & \geqq\left[\frac{1}{4}-\frac{\varrho}{2 q^{2}}(j+1)\right] x^{4 q+4}-\left[\frac{15}{2}+\frac{1}{2 \varrho}(j+1)\right] q^{2} x^{4 q} \\
& \geqq\left[\frac{1}{4}-\frac{\varrho}{2}(j+1)\right] x^{4 q+4}-\left[\frac{15}{2}+\frac{1}{2 \varrho}(j+1)\right] q^{2} x^{4 q} .
\end{aligned}
$$

Denoting by $\Delta(x)$ the difference between the left hand side and the right hand side of Eq. (III.21), we deduce from Eqs. (A.9) and (A.12)

$$
\begin{aligned}
\Delta(x) \geqq & {\left[\frac{1}{4}-\frac{\varrho}{2}(j+1)-a^{2}\right] x^{4 q+4}-\left[\frac{15}{2}+\frac{1}{2 \varrho}(j+1)\right] q^{2} x^{4 q}+\frac{a^{2}}{4}(c q)^{2 q} x^{4} } \\
& -a^{2}(c(n-1))^{2(n-1)} x^{4 m+8}
\end{aligned}
$$

or, by putting $y \equiv x^{4} / c^{2} q^{2}$ :

$$
\Delta(x) \geqq \frac{a^{2}}{4}(c q)^{2 q+2} y \delta(y),
$$

with

$$
\begin{aligned}
\delta(y) \equiv & {\left[\frac{1-2 \varrho(j+1)}{a^{2}}-4\right] y^{n+m}-\frac{1}{a^{2} c^{2}}\left[30+\frac{2}{\varrho}(j+1)\right] y^{n+m-1} } \\
& +1-4\left(\frac{n-1}{n+m}\right)^{2(n-1)} y^{m+1} .
\end{aligned}
$$

The truth of inequality (III.21) now depends on whether the polynomial $\delta(y)$ can be made positive for $y \geqq 0$ by some $(n, m)$-independent choice of the two free parameters $\varrho$ and $c$. It turns out that this is achieved by so adjusting $\varrho$ and $c$ as to equalize (in absolute value) the first three coefficients of $\delta(y)$, namely by choosing $\varrho=\left(1-5 a^{2}\right) / 2(j+1)$ and :

Indeed, we have then

$$
c^{2}=\frac{1}{a^{2}}\left[30+\frac{4(j+1)^{2}}{1-5 a^{2}}\right]
$$

$$
\delta(y)=y^{n+m}-y^{n+m-1}+1-4\left(\frac{n-1}{n+m}\right)^{2(n-1)} y^{m+1} .
$$


For $n=1$, the last term is in fact absent, so that

$$
\delta(y)=y^{m+1}-y^{m}+1 \begin{cases}=y \geqq 0 & \text { if } m=0, \\ \geqq\left[1-\frac{m^{m}}{(m+1)^{m+1}}\right]>0 & \text { if } \quad m \geqq 1 .\end{cases}
$$

For $n \geqq 2$, noticing that $4[(n-1) /(n+m)]^{2(n-1)} \leqq 1 /(m+1) \forall m=0,1, \ldots$, we obtain

$$
\begin{aligned}
\delta(y) & \geqq y^{n+m}-y^{n+m-1}+1-\frac{1}{m+1} y^{m+1} \\
& \geqq y^{n+m}-y^{n+m-1}-\frac{1}{m+1} y^{m+1}+\frac{1}{m+1} \\
& =(y-1)^{2}\left[y^{m}\left(1+y+\ldots+y^{n-2}\right)+\frac{1}{m+1}\left(1+2 y+\ldots+m y^{m-1}\right)\right] \geqq 0,
\end{aligned}
$$

and the proof is complete.

Acknowledgements. I am grateful to G. Mennessier for his interest and his careful reading of the manuscript. I also wish to thank T. Boissière and G. Voisin for useful discussions.

\section{References}

1. Graffi, S., Grecchi, V., Simon, B.: Phys. Lett. 32B, 631 (1970)

2. Eckmann, J.P., Magnen, J., Sénéor, R.: Commun. Math. Phys. 39, 251 (1975) Magnen, J., Sénéor, R.: Commun. Math. Phys. 56, 237 (1977)

3. Risken, H., Vollmer, H.D. : Z. Phys. 201, 323 (1967)

Haken, H. : Laser theory. In : Encyclopedia of Physics, Vol. XXV/2c. Princeton N.J. : Van Nostrand 1970

4. Kaushal, R.S.: J. Phys. A12, L 253 (1979)

5. Mitra, A.K.: J. Math. Phys. 19, 2018 (1977)

6. Bender, C., Wu, T.T.: Phys. Rev. Lett. 21, 406 (1968); Phys. Rev. 184, 1231 (1969)

7. Auberson, G., Mennessier, G.: In preparation

8. Kato, T.: Perturbation theory for linear operators, Chap. VIII. Berlin, Heidelberg, New York: Springer 1966

9. Simon, B.: Ann. Phys. 58, 76 (1970)

10. Simon, B.: Adv. Math. 7, 240 (1971)

11. Reed, M., Simon, B.: Methods of modern mathematical physics

a) Vol. I (Functional analysis). New York, London: Academic Press 1972

b) Vol. IV (Analysis of operators), Chap. XII. New York, San Francisco, London: Academic Press 1978

12. Ref. [8, Chap. V, Sect. 3.10]. See also Ref. [11a, Chap. VIII, Sect. 6]

13. Ref. [11a, Appendix of Chap. V, Sect. 3] and Ref. [9, Lemma II.11.1]

14. Auberson, G., Mennessier, G. : J. Math. Phys. 22, 2472 (1981)

15. Watson, G.N.: Philos. Trans. R. Soc. (London) Ser. A 211, 279 (1912)

Hardy, G.H. : Divergent series. Oxford: University Press 1949

16. Boissière, T.: Private communication

17. Loeffel, J.J., Martin, A., Wightman, A.S.: Phys. Lett. 30B, 656 (1969)

See also Loeffel, J.J., Martin, A. : Prétirages de la RCP n 25. R. Gerard (ed.). Strasbourg (1970)

18. Ref. [9, Theorem II.3.1]

Communicated by J. Ginibre

Received November 16, 1981 